\title{
Idhini fahamishi katika majaribio ya kikliniki ya ukingaji wa virusi vya ukimwi
}

Population Council

Follow this and additional works at: https://knowledgecommons.popcouncil.org/departments_sbsr-hiv

Part of the Demography, Population, and Ecology Commons, Family, Life Course, and Society Commons, Gender and Sexuality Commons, International Public Health Commons, and the Medicine and Health Commons How does access to this work benefit you? Let us know!

\section{Recommended Citation}

"Idhini fahamishi katika majaribio ya kikliniki ya ukingaji wa virusi vya ukimwi." New York: Population Council, 2008. 


\section{IDHINI FAHAMISHI}

KATIKA MAJARIBIO YA KIKLINIKI YA UKINGAJI WA VIRUSI VYA UKIMWI

BAADHI YA MASWALA YALIYOANGAZIWA KUTOKA KATIKA WARSHA YA KIMATAIFA 


\section{IDHINI FAHAMISHI}

KATIKA MAJARIBIO YA KIKLINIKI YA UKINGAJI WA VIRUSI VYA UKIMWI

BAADHI YA MASWALA YALIYOANGAZIWA KUTOKA KATIKA WARSHA YA KIMATAIFA 
Haki ya Kunakili @ 2008 The Population Council, Inc.

\section{(2) Population Council}

The Population Council inaendesha utafiti kote duniani kuboresha sera, programu, na bidhaa katika mada tatu: Virusi vya Ukimwi na UKIMWI; umaskini, jinsia, vijana; na afya ya uzazi.

One Dag Hammarskjold Plaza

New York, NY 10017 USA

Simu: +1-212-339-0500

Kipepesi: +1-212-755-6052

\section{Shukurani}

Warsha juu ya idhini fahamishi katika majaribio ya ukingaji wa Virusi vya Ukimwi, iliyofanyika 16-18 Mei 2005 jiijini New York, ilibuniwa na kupangwa na kamati iliyokuwa na Barbara Friedland, Martha Brady, na Hillary Bracken (Population Council), Kathleen MacQueen (Family Health International), Cynthia Woodsong (Research Triangle Institute), na Elizabeth McGrory (mshauri). Warsha ilifanywa kwa pamoja na Population Council na Family Health International na ilisaidiwa na Shirika la Misaada la Marekani (USAID), Ubia wa Kimataifa wa Microbicides, Inc., Taasisi za Kitaifa za Afya, na Wakfu wa William na Flora Hewlett.

Tafsíri na: Samwel Oyugi Isaac

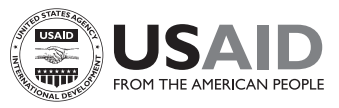

Taarifa hii imefanikishwa kwa usaidizi mkarimu wa Wamarekani, kupitia kwa Shirika la Misaada la Marekani (USAID). Yaliyomo katika taarifa hii ni jukumu la kipekee Population Council na kamwe hayaakisi maoni ya USAID au Serikali ya Marekani.

Sehemu yoyote ya taarifa hii inaweza kunakiliwa au kutoholewa kutosheleza viwango vya mahali pale pale bila ruhusa kutoka kwa Population Council, alimradi kuwa sehemu zilizonakiliwa zimesambazwa bure au kwa gharama (sio kwa faida) na kuwa chanzo kitatambulishwa. Utoaji wowote wa kibiashara unahitaji ruhusa ya awali kutoka kwa Population Council. Population Council litashukuru kupokea nakala ya nyenzo zozote zinazorejelea matini. 


\section{Yaliyomo}

Utangulizi

Zana za idhini fahamishi: Kuelezea dhana ngumu

Vijitabu

Video

Elimu ya mshiriki yenye msingi wa taralikishi wasilianifu

Kueleza dhana ngumu

Kukadiria ufahamu miongoni mwa washiriki wa jaribio:

$\mathrm{Ni}$ nini washiriki wanahitaji kujua?

Umuhimu wa mafunzo

Hitimisho

Kiambatisho 1: Mitazamo ya kupima kiasi na kuelezea ubora

Kiambatisho 2: Jaribio la HPTN 035: Orodha kaguzi ya ufahamu wa kuandikisha idhini fahamishi (Imedondoolewa) 


\section{Utangulizi}

Tatika uwanja unaobadilika wa majaribio ya kikliniki ya ukingaji wa Virusi vya I Ukimwi, idhini fahamishi inatambulika kama kipimo cha kihakiki cha kufanya maadili yaendeshwe. Tunapohakikisha idhini fahamishi na ushiriki wa kujitolea ni mojawapo ya vipengele tatanishi zaidi vya jaribio lolote la kikliniki, majaribio ya ukingaji wa Virusi vya Ukimwi, mengi ambayo yanaendeshwa katika mazingira yenye umaskini wa rasilimali, na hivyo basi kutoa changamoto zaidi za kimaadili na kiutendaji:

- Majaribio ya kiwango kikubwa ni lazima yaendeshwe katika maeneo yenye athari ya juu ya Virusi vya Ukimwi.

- Majaribio yanahitaji watu wanaojitolea wenye afya, ambao mara nyingi hudhuriwa kiuchumi na kijamii na katika hatari kubwa ya kuambukizwa Virusi vya Ukimwi.

- Watafiti lazima wawakumbushe washiriki kuwa bidhaa inayotathminiwa bado haijathibitishwa kufanya kazi ili washiriki wasihisi hisi ya kinga ya uwongo ambao inaweza kusababisha ongezeko la mienendo hatari (kupunguza utumiaji wa kondomu au kuongeza idadi ya washikaji).

- Majaribio ya kuzuia Virusi vya Ukimwi/UKIMWI yanahusisha maswala nyeti ya hali ya juu: waa, ujinsia na mahusiano ya kijinsia.

- Ufikishaji wa istilahi za kiteknolojia za utafiti na dhana zisizojulikana katika lugha za mahali pale pale kunaweza kutatiza.

Changamoto hizi zinazidishwa katika mandhari/mazingira yenye kiwango cha chini cha kujua kusoma na kuandika, pale ambapo washiriki wakuu katika utafiti wanaweza kuwa na ufahamu mdogo wa afya ya jinsia au utafiti wa kikliniki.

Ili kushughulikia changamoto hizi, Population Council na Family Health International (FHI) yaliitisha mkutano kutambulisha mitazamo ya kufanikiwa ya mchakato wa idhini fahamishi ambayo ingeweza kutoholewa na kutumiwa kwa pamoja miongoni mwa watafiti wanaotekeleza majaribio ya uzuiaji wa Virusi vya Ukimwi. Mukhtasari huu, umetoholewa kutoka kwa ripoti kamili ya mkutano, ${ }^{1}$ unalenga zana vumbuzi na mikakati inayotumika kusahalisha idhini fahamishi katika majaribio ya uzuiaji wa Virusi vya Ukimwi, na kushirikisha mifano yenye ufafanuzi kutoka kwa majaribio yaliyokamilika na yanayoendelea.

\footnotetext{
${ }^{1}$ C. Elizabeth McGrory, Barbara A. Friedland, Cynthia Woodsong, na Kathleen M. MacQueen. 2006. Informed Consent in HIV Prevention Trials: Report of an International Workshop. New York: Population Council na Family Health International. Yapatikana katika: www.popcouncil.org/pdfs/ICWorkshop.pdf.
} 


\section{Zana za idhini fahamishi: Kuelezea dhana ngumu}

VX achunguzi katika idadi ya tafiti za hivi sasa za uzuiaji wa Virusi vya Ukimwi wanatumia zana bunifu na mitazamo kuboresha ufahamu wa wanaojitolea kwenye utafiti wa dhana za utafiti na taratibu za jaribio, pamoja na vijitabu vya mafunzo, karatasi zenye taarifa ya kweli, chati za kugeuzwa, video, vielelezo, na elimu shiriki yenye msingi wa taralikishi wasilianifu. ${ }^{2}$

\section{Mapendekezo ya kukuza vifaa vya idhini fahamishi na mitazamo}

- Endesha utafiti muundo kwa uangalifu: rasimu, tathmini awali, na tohoa kwa mazingira ya mahali pale pale.

- Husisha wafanyikazi wa utafiti, wanachama wa bodi ya ushauri wa kijamii, wanaoweza kuwa wasiriki, na, iwapo itawezekekana, washiriki kutoka katika tafiti za awali katika kuzalisha mawazo, kukuza mitazamo, na kutathmini awali nyenzo.

- Tumia bodi za ushauri wa kijamii na miundo mingine ya kijamii kuimarisha dhana muhimu, kama kujitolea, na kusaidia katika ukuzaji lugha, istilahi na analojia ambazo zinafikisha dhana za utafiti ambazo zinahusiana na kueleweka katika mazingira ya mahali pale pale.

- Tumia nyenzo nyingi kwa pamoja kuchangia na kuimarishana katika kupitisha taarifa zinazohusika kwa kijamii na washiriki kotekote katika mchakato wa jaribio la kikiliniki.

${ }^{2}$ Teknolojia wasilianifu ni pamoja na ACASI—tarakilishi sikivu- inayosaidia "mafunzo" nafsi au "mahojiano"nafsi-aidha hutumiwa kutoa taarifa kwa wanaojitolea kwenye utafiti au kama mbinu ya kuhoji kushawishi majibu juu ya maswala nyeti. 


\section{Vijitabu}

Tijitabu kilichotengenezwa vizuri kinaweza kuwa kifaa kizuri cha kuonyesha L weledi wa jaribio na heshima kwa washiriki. Vielelezo mifano hai (halisi) vya rangi vingesaidia washiriki kuelewa na kukumbuka taarifa. Vijitabu vya utafiti/ jaribio vinaweza kuwa na manufaa kwa:

- Kupitisha taarifa za kawaida au za kinaganaga kwa washiriki wa utafiti juu ya hatua na taratibu za utafiti na kunyongezea fomu za idhini fahamishi;

- Kufasili na kueleza kwa mifano iliyoandikwa au iliyochorwa dhana za utafiti zisizoeleweka kwa urahisi.

- Kujibu maswali wakati wa vikao vya kuarifu.

- Kusaidia katika uandikishaji iwapo washiriki wataonyeshana vijitabu kwa marafiki au jamaa.

\section{Zana za idhini fahamishi katika matumizi: Population Council Awamu ya 3 jaribio} la Carraguard ${ }^{\circledR}$

Kijitabu kilichochapishwa na Population Council cha jaribio la microbicide ${ }^{3}$ awamu ya 3 la Carraguard ${ }^{\circledR}$ kilinyongezea fomu ya idhini fahamishi. Wakati wa kutathmini awali, washiriki walitoa maoni juu ya picha, rangi, na ubora wa ujumla wa kijitabu, kulikosababisha mabadiliko ya kisanifu. (tazama kijisanduku)

\section{Zana za idhini fahamishi katika matumizi: HPTN 035 jaribio la microbicide}

Kijitabu cha Mtandao wa Majaribio ya Uzuiaji wa Virusi vya Ukimwi (HPTN) 035 jaribio la microbicide ${ }^{4}$ kinatumia mchoro wa hulka aliyejulikana kama Serena kuelezea dhana tofauti za utafiti na taratibu. Jaribio liliendeshwa katika maeneo mengi katika nchi kadhaa; hivyo basi nyenzo zilifanyiwa jaribio tathmini na kutoholewa ili kutumika kwa kila mazingira. Ingawaje Serena alisanifishwa kimakusudi kuonekana mwenye kujulikana kwa wanawake wanaoandikishwa kwa jaribio, tathmini awali ilikuwa msingi wa Serena kuchorwa upya kuonekana wa kisasa sana, anayevutia, na mwenye ukwasi. Zaidi ya hayo, mchoro mmoja wa Serena ulifafanuliwa/ulieleweka na wengi wa wanaoweza kuwa washiriki kama anaonekana kuwa na wasiwasi hivyo basi mchoro huo ulichorwa tena baadaye. (tazama kijisanduku)

\footnotetext{
${ }^{3}$ Jaribio la awamu ya 3 ya usalama na matokeo ya kufaa ya microbicide Carraguard kukinga seroconversion ya Virusi vya Ukimwi katika wanawake liliendeshwa katika maeneo matatu nchini Afrika Kusini kati ya Machi 2004 na Machi 2007. www.popcouncil.org/projects/MIC_CarraClinicalStudies3.html. Ilifikiwa 17 Januari 2008 .

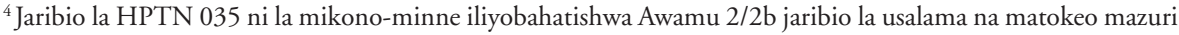
ya microbicides mbili mtahiniwa na mikono miwili thibiti iliyoandikisha wanawake 3,220 katika maeneo manane katika nchi tano. www.hptn.org/research_studies/hptn035.asp; www.mtnstopshiv.org/node/62. Ilifikiwa 17 Januari 2008.
} 
Marekebisho ya kijitabu cha Carraguard Awamu ya 2 kwa Awamu ya 3

Awamu ya 2

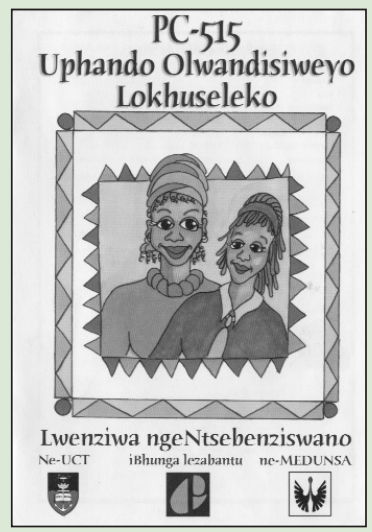

Awamu ya 3

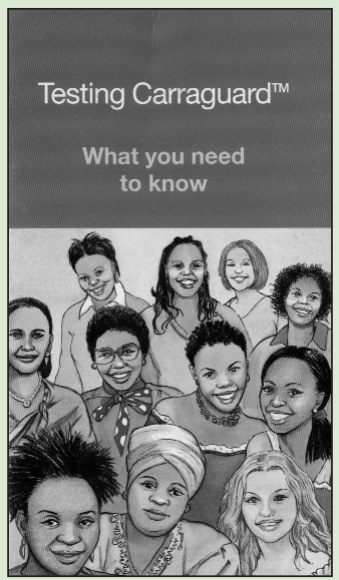

Washiriki katika jaribio la Carraguard Awamu ya 2 walipendekeza kuwa vielelezo kwa kijitabu cha taarifa (kushoto) viwe na uhalisi zaidi. Mabadiliko yalifanyiwa kijitabu cha Carraguard Awamu ya 3 (kulia).

\section{Mabadiliko ya "Serena” kwa HPTN 035}
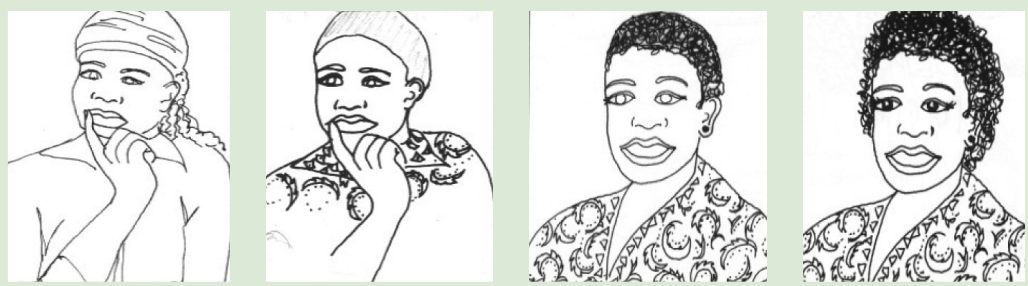

Katika tathmini awali, washiriki walisaidia kusahilisha Serena, iliyotumika katika vijitabu vya jaribio. (Usanii na Denise Todloski) 


\section{Video}

Tideo zinaweza kusahalisha mchakato wa uandikishaji na kusahalisha majadiliano ya maana juu ya idhini fahamishi, hasa katika majaribio ya viwango vikubwa. Video zinaweza kuwa za kuhusisha zaidi ikilinganishwa na habari zilizoandikwa, zinaweza kuwa kifaa muhimu katika mazingira yenye hali ya chini kimasomo, na zinaweza kutumika katika kusanifisha utoaji wa taarifa juu ya jaribio na kupunguza mzigo kwa wafanyikazi wa utafiti.

Changamoto za teknolojia ya video ni pamoja na:

- Una mchakato mgumu wa ukuzaji na unahitaji muda mrefu wa kuandaa; hii inamaanisha ni vigumu kuridhia mabadiliko ya hatua za utafiti katika video wakati wa utoaji.

- Kuonyesha video katika maeneo yote hakutawezekana kwa kuwa kuleta vifaa ghali katika maeneo ya mahali pale pale kunaweza kuleta usumbufu au hata hatari.

- Kuvutia hisia za wanaoweza kuwa washiriki katika kliniki zenye shughuli nyingi kunaweza kuwa kugumu.

Zana za idhini fahamishi katika matumizi: Utafiti wa MIRA wa kiwambo cha mpira (kuzuia mimba) kuzuia uambukizaji wa Virusi vya Ukimwi katika wanawake Wafanyikazi walioshiriki katika jaribio la MIRA ${ }^{5}$ waligundua kuwa washiriki walionekana kuchoshwa na nyenzo zilizoandikwa au kwa kuwasikiza wafanyikazi wakiwasilisha taarifa; wanaojitolea walionyesha kuwa video ingesaidia katika kuwashughulisha.

- Wafanyikazi katika kliniki, wanachama wa kijamii, na timu ya utafiti walishughulishwa katika ukuzaji wa video.

- Washiriki wa jaribio waliitikia kihalisi kwa umbo la video ya mkuu wa kamati ya maadili(walipendezwa kuona kuwa kiongozi wa kamati ya maadili ameonyeshwa kwenye video) kwa sababu:

-Ilisaidia kutoa sura halisi kwa dhana ya 'upitiaji upya wa maadili'

-Ilituma ujumbe kuwa washiriki wanadhaminiwa;

-Ilifikisha weledi wa utafiti (ilionyesha kuwa utafiti ulikuwa wa hali ya juu)

\footnotetext{
${ }^{5}$ Jaribio la MIRA (Njia za Kuboresha Afya ya Uzazi katika Afrika) linaendeshwa Durban na Johannesburg, Afrika Kusini, na Harare, Zimbabwe.

www.cervicalbarriers.org/documents/MIRA_Results_and_Publications_aug07_final_pdf.pdf. Ilifikiwa 17 Januari 2008.
} 


\section{Zana za idhini fahamishi katika utendaji: Population Council Awamu ya 3 jaribio la Carraguard ${ }^{\circledR}$}

Ili kuboresha mitazamo zana zilizotumika katika awamu ya 3 ya Carraguard $^{\circledR}$ nchini Afrika Kusini, Population Council liliendesha majadiliano ya kundi lengwa na mahojiano ya kina na washiriki na wafanyikazi wa jaribio la awamu ya $2 .{ }^{6}$ Population Council lilitafuta kutambulisha njia za kuelezea dhana ngumu na liliomba mapendekezo ya kuandikisha na mifuatano ya idhini fahamishi kwa jaribio la awamu ya 3. Kwa msingi wa majibu, Population Council lilikuza video ${ }^{7}$ ya kuelezea wazi dhana zisizoeleweka na kuwasilisha taarifa za msingi kwa njia ihusishayo zaidi.

- Hadithi fupi zilitumika kufikisha dhana ngumu na wazo kuu kama ubahatishaji, upofushaji maradufu(mtafiti na mshiriki wote hawajui kama mshiriki amewekwa katik kundi kwa kutumia bidhaa ya utafiti au kipozauongo), ukushiriki wa kujitolea, kuwasiliana na mshikaji, uchunguzi wa fupanyonga (utaratibu usiojulikana kwa wanawake wengi katika jamii za jaribio), na upimwaji wa Virusi vya Ukimwi.

- Mshauri wa kielimu alisaidia kukuza mswada, na mwigizaji wa kike anayejulikana alitokeza kama msimulizi kwenye kamera.

- Sehemu za video zilifanyiwa tathmini ya awali na kutoholewa kufuatia mijadala na kundi lengwa la wanawake katika jamii za jaribio pamoja na wanachama wa bodi ya ushauri wa kijamii.

- Ripoti za mchapo zinapendekeza kuwa wafanyikazi wa utafiti na washiriki wa jaribio walipata video kuwa ya kuarifu na kuvutia; wanawake walishukuru kuona mapema yale ambayo wangeyapitia katika jaribio.

\footnotetext{
${ }^{6}$ A. De Kock, M. Marumo, na B. Friedland. 2005. "Carraguard Phase 3 Trial: Developing and implementing a video." Wasilisho katika warsha ya Population Council/Family Health International juu ya majaribio ya Idhini Fahamishi katika Uzuiaji wa Virusi vya Ukimwi, New York, 16-18 Mei.

7 “Testing Carraguard ${ }^{\mathrm{TM}}$. What you Need to Know." Video. Johannesburg: Population Council, 2004. Imetengenezwa na Pandamonium Productions. www.popcouncil.org/media/saclips/videolink.html. Ilifikiwa 17 January 2008.
} 


\section{Elimu ya mshiriki yenye msingi wa taralikishi wasilianifu}

Zana za idhini fahamishi katika matumizi: Mradi wa BOTUSA jaribio la PrEP

Mradi wa BOTUSA jaribio la $\operatorname{PrEP}^{8}$ uliwasilisha mpango wa kutumia tarakilishi wasilianifu inayosaidia mafunzo nafsi baada ya mchakato wa nguvu wa ana kwa ana wa kuidhinisha na kabla ya uendeshaji tathmini ya ufahamu. Teknolojia hii inatumia tarakilishi ya skrini gusa yenye sehemu ya kusikia, na taarifa juu ya jaribio zinawasilishwa katika uwasilisho wa medianuwai ulio na usimulizi mwandamizi; washiriki wana hiari ya kuchagua lugha.

${ }^{8}$ Jaribio la PrEP Botswana, utafiti wa kutathmini usalama na kufaa kwa ukingaji kabla ya kuambukizwa (preexposure prophylaxis) katika kupunguza uambukizaji wa Virusi vya Ukimwi miongoni mwa vijana wanaovutiwa na jinsia tofauti, inaendeshwa na mradi wa BOTUSA — ushirikiano wa Serikali ya Botswana na Vituo vya Marekani vya Kuthibiti na Kukinga Maradhi (CDC). www.cdc.gov/hiv/resources/factsheets/prep.htm. Ilifikiwa 17 Januari 2008. 


\section{Kueleza dhana ngumu}

Ti vigumu sana kuelezea dhana za majaribio ya kikliniki na hatua za utafiti kwa 1 wanaojitolea kwenye utafiti katika mazingira ya namna mbalimbali na lugha mbalimbali kwa njia ambayo inahakikisha kuwa wanaweza kufahamu. Wafanyikazi wa jaribio wanashughulisha mitazamo vumbuzi kuwasilisha na kuelezea dhana ngumu katika nyenzo za idhini fahamishi ambazo zinazotumika katika mazingira ya mahali pale pale. ${ }^{9,10}$

Mifano ya njia vumbuzi za kuelezea dhana ngumu zilizojadiliwa katika mkutano ni pamoja na:

Ubahatishaji: Huu ni utaratibu wa kugawa washiriki katika makundi tibiwa kwa ugawaji nasibu, ili kuondoa uchaguzi wa upendeleo wa watafiti au washiriki, kuhakikisha kuwa kuna uchanganuzi halali wa takwimu za data zilizokusanywa, na kwa kuunda makundi yanayotangamana kwa kuzingatia vipengele ambavyo vinaweza kuathiri matokeo. Ubahatishaji ni dhana ngumu ya utafiti ambayo inaweza kuwa ya kutofahamika wazi kwa wanaoweza kuwa washiriki na ni vigumu kuelezea kwa njia ile ile katika mazingira mbalimbali:

- Ubahatishaji mara nyingi huelezwa kwa kutumia picha zinazohusishwa na wazo la nasibu, kama mashine ya bahati nasibu au kubingirisha dadu (tazama kijisanduku).

- Picha ya tarakilishi inaweza kutoa hoja kuwa programu ya tarakilishi, badala ya mtu, kwa nasibu inateua washiriki wa jaribio kwenye kundi ingiliano au dhibiti.

\section{Ubahatishaji}
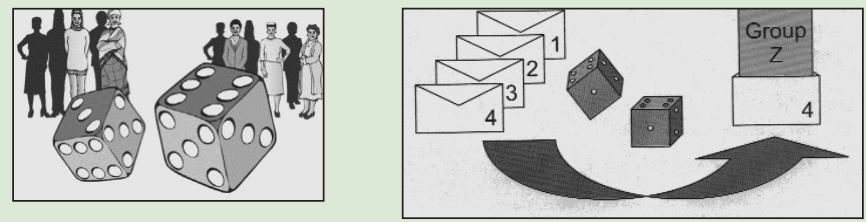

Picha za vitu vinavyohusishwa na wazo la nasibu—kubingirisha dadu—zilitumika kuelezea kuwa washiriki wanachaguliwa kwa kubahatisha kwa mwingiliano wa utafiti na makundi thibiti. Kushoto, kielelezo kutoka kwa jaribio la kiklininki Awamu ya 3 ya Carraguard; kulia kutoka kwa jaribio la PrEP la BOTUSA.

\footnotetext{
${ }^{9}$ H. Bracken. 2005. "Communicating complex concepts." Wasilisho katika warsha ya Population Council/Family Health International juu ya majaribio ya Idhini Fahamishi katika Uzuiaji wa Virusi vya Ukimwi, New York, 16-18 Mei.

${ }^{10}$ Kwa sababu athari nzuri ya njia hizi kwa kufikisha taaarifa kwa washiriki wa jaribio haijatathminiwa hatua kwa hatua, zingechukuliwa mapendekezo badala ya "desturi bora kabisa."
} 
Kipozauongo: Maelezo yanasisitiza kuwa bidhaa tafiti na kipozauongo zinafanana isipokuwa kipozauongo:

- Si "tendaji," au "haina [jina la kiambato tendaji] ndani yake"

- "Moja ina [kiambato tendaji] na nyingine haina"

- Ni dutu kama jeli ya kulinganisha (tazama kijisanduku)

Njia zingine za kuelezea kipozauongo ni pamoja na picha za kulingana ambazo lazima zichukuliwe kutoka kwa na kutathminiwa katika muktadha wa mahali pale pale ili mfano uwe na uhusiano.

- Maji ya matunda au maziwa yaliyotajirishwa Vitamini.

- Vito bandia dhidi ya johari halisi.

- Kola bandia dhidi ya Koka-Kola ${ }^{\text {Tm }}$

Serodiscordance (Hali ya mshikaji mmoja kuwa HIV chanya na mwingine HIV hasi): Inaweza hasa kuwa vigumu kuelezea dhana ya kuwa mshikaji mmoja anaweza kuwa na Virusi vya Ukimwi na mwingine asiwe navyo. Kuhimiza wachumba kupimwa Virusi vya Ukimwi, Washirika katika Uzuiaji jaribio la HSV/HIV ${ }^{11}$ wanatumia analojia ya miti ya mibuyu iliyoambukizwa na mchwa (tazama kijisanduku). Mtu hawezi kusema kutoka mbali mti upi una mchwa wala mshikaji yupi ana Virusi vya Ukimwi bila kupima. Analojia ya mti wa mbuyu itafaa katika mazingira mengine lakini haitahusiana katika mengine, ikielezea umuhimu wa kutathmini awali na kutohoa picha na lugha katika kila mazingira kuhakikisha kuwa zinafikisha ujumbe husika na kusahalisha ufahamu.

Maswala nyeti: Ujinsia na maswala mengine nyeti yanashughulikiwa kwa kupitia aidha picha za wazi wazi au za kupendekeza (tazama kijisanduku):

- Nyenzo zingine hutumia michoro kama viatu ama nguo kando ya kitanda (bila kuonyesha watu) kuashiria kitendo cha ngono.

- Wengine hutumia michoro dhahiri ya mwanaume uchi na mwanamke waliolala pamoja.

- Vivyo hivyo, uchunguzi wa fupanyonga unaweza kuashiriwa kwa michoro ya wazi wazi au zenye uwazi mdogo.

- Jaribio moja ni pamoja na vielelezo vya uchunguzi wa fupanyonga tu katika nyenzo zinazotumika katika kliniki lakini si katika nyenzo ambazo washiriki huchukua nyumbani. Hivi ni kusuluhisha tatizo kuwa michoro ya wazi ikionekana na watu wengine kuweza kutia waa utafiti au washiriki.

\footnotetext{
${ }^{11}$ Jaribio la Awamu ya 3 ya acyclovir kwa HSV-2 ukandamizaji miongoni mwa wachumba wasioelewana wenye Virusi vya Ukimwi, inayoendeshwa katika maeneo mengi Afrika na mradi wa Washirika katika Uzuiaji (Chuo Kikuu cha Washington). http://clinicaltrials.gov/show/NCT00194519;

http://depts.washington.edu/hsvhiv/public/index.html. Ilifikiwa 17 Januari 2008.
} 


\section{Kipozauongo}

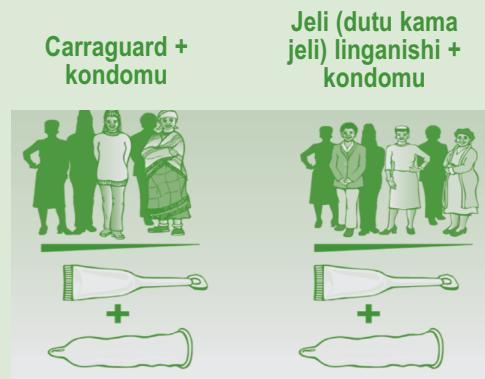

Kuhuisha katika video ya Carraguard Awamu ya 3 kulisisitiza kuwa bidhaa na kipozauongo vinafanana na vinatumika kwa njia sawa.

\section{Uchunguzi wa fupanyonga}
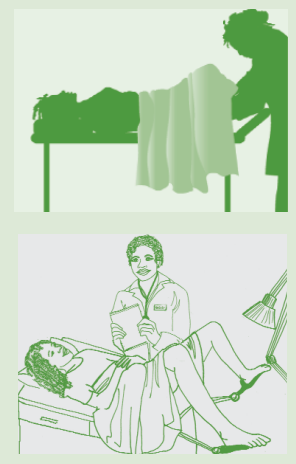

Washiriki wengine walidhihirisha kuwa kuwa wazi sana katika nyenzo ambazo zinaweza kuchukuliwa nyumbani au kuonekana na watu wengine kunaweza kutia waa utafiti au washiriki. Picha ya juu kutoka kwa jaribio la PrEP Mradi wa BOTUSA; chini kutoka HTPN.

\section{Serodiscordance}

(Hali ya mshikaji mmoja kuwa HIV chanya na mwingine HIV hasi)
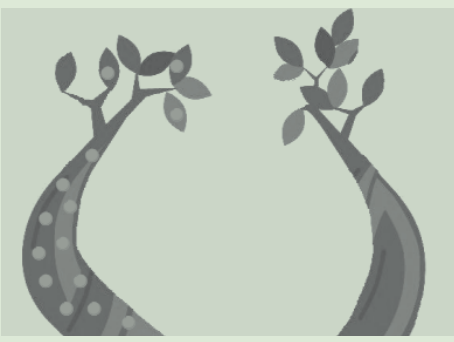

Miti miwili ya mibuyu inaonekana kufanana ingawaje mmoja umeambukizwa na mchwa ambao wanaweza kuharibu mti mmoja au yote. Kielelezo kilichotumika ni analojia ya Virusi vya Ukimwi, ambavyo vinaweza kuambukiza mshikaji mmoja au wote lakini haviwezi kubainishwa bila upimaji. Kielelezo kutoka kwa Washirika katika Uzuiaji jaribio la HSV/HIV.

\section{Shughuli za kingono}

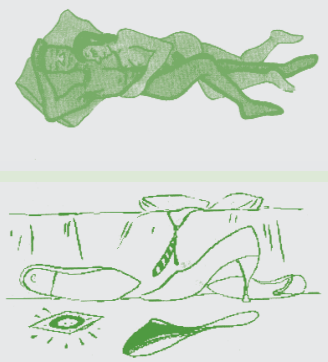

Kutegemea eneo la utafiti, nyenzo katika baadhi ya majaribio ya Carraguard yalitumia michoro/picha za wazi (juuAfrika Kusini) au zinazopendekeza (chini-Thailand) kupendekeza ngono. 


\section{Kukadiria ufahamu miongoni mwa washiriki wa jaribio: Ni nini washiriki wanahitaji kujua?}

Ti kuboresha idhini fahamishi, wafanyikazi wengi wa jaribio wanajaribu kuamua I namna ya kupima ufahamu wa washiriki wa maswala ya jaribio. Watafiti wanaosanifu ukadiriaji wa idhini fahamishi wanakabiliwa na chaguo la kama washughulishe mtazamo kiasi au ubora (tazama Kiambatisho 1).

- Ufahamu wa dhana mahususi na utaratibu wa utafiti ungechukuliwa kuwa muhimu sana, kiasi cha kwamba wanojitolea lazima waonyeshe ufahamu wa hali ya juu kabla ya kushiriki?

- Ni njia zipi zingetumika kukadiria kiwango cha ufahamu?

Ingawa ufahamu kamili wa vidokezi vyote vya jaribio ni lengo muhimu la idhini fahamishi, hata hivyo hili mara nyingi halitimiziki. Watafiti wengi walizingatia idadi fulani ya maswala kuwa ya umuhimu kwa wanaojitolea kwenye jaribio kufahamu:

- Dhamira ya utafiti.

- Kuwa utafiti ni wa majaribio.

- Tashwishi juu ya matokeo ya kufaa ya bidhaa tathmini katika ukingaji wa uambukizaji wa Virusi vya Ukimwi.

- Haja ya kuendelea na desturi zingine zinazosaidia kupunguza hatari wakati wa kushiriki katika utafiti.

- Dhana ya ubahatishaji, kupofusha, na vipozauongo.

- Utaratibu wa jaribio, hatari, faida, ujitoleaji na usiri.

Tazama Kiambatisho 2 kwa mfano wa zana ya ukadiriaji wa ufahamu wa HPTN. 


\section{Umuhimu wa mafunzo}

工 ata kama kuna vifaa vizuri, nyenzo na ukadiriaji zitakuwa na matumizi yenye 1 mipaka mpaka pale wafanyikazi wa utafiti watapewa mafunzo na usaidizi wa kuvitumia ifaavyo na kwa njia ile ile. Kukuza na kutumia mitazamo ya kufikisha maswala yasiyoeleweka kuboresha idhini fahamishi na kwa kutathmini matokeo ya kufaa (kufanya kazi kwao), kunaweza kuwa na gharama na kuhitaji ufadhili na kujitolea kwa wadhamini wa jaribio, wafadhili na timu za jaribio. Rasilimali za kutosha lazima zitengwe kando katika bajeti za jaribio ya mafunzo yanayoendelea na tathmini.

\section{Hitimisho}

W atafiti na wafanyikazi wa utafiti katika mazingira ya aina zote wanastahili kuchangia mikakati na zana na kupendekeza njia za kuongeza kiwango cha ufahamu wa washiriki ili kuboresha idhini fahamishi. Kwa kuongezea uimarishaji wa ushuhuda wa uwekezaji ulioongezeka, mabadilishano kama haya yanaweza pia kusuluhisha changamoto, pamoja na kutoa maamuzi juu ya njia za mawasiliano na mikakati zipi za kutumiwa, mafunzo na mitazamo mahususi ya kuelezea dhana zisizoeleweka. 


\section{Kiambatisho 1: Mitazamo ya kupima kiasi na kuelezea ubora}

\begin{tabular}{|c|c|c|}
\hline & Nguvu & Udhaifu \\
\hline $\begin{array}{l}\text { Mitazamo } \\
\text { inayopima } \\
\text { kiasi } \\
\text { (maswali } \\
\text { yaliyofungwa, } \\
\text { kweli/si kweli, } \\
\text { kuchagua kutoka } \\
\text { kwa orodha ya } \\
\text { majibu) }\end{array}$ & $\begin{array}{l}\text { - Nzuri kwa kupata taarifa } \\
\text { mahususi, majibu ya neno } \\
\text { moja, au kwa kupeleleza } \\
\text { ukweli. } \\
\text { - Ni rahisi kuendesha } \\
\text { - Tathmini ni madhumuni } \\
\text { kiasi. } \\
\text { - Inahitaji mafunzo } \\
\text { machache ya wafanyikaz }\end{array}$ & $\begin{array}{l}\text { - Ina mipaka kwa kukadiria ufahamu wa dhana } \\
\text { ngumu } \\
\text { - Zinaweza kutoa wazo kuwa mshiriki ameelewa, } \\
\text { lakini hupima tu uwezo wa muda mfupi wa } \\
\text { kurudia taarifa. } \\
\text { - Uchaguzi wa maneno mahususi/ istilahi tata } \\
\text { katika majaribio ya maeneo mengi/ lugha nyingi. } \\
\text { - Maswali ya kweli/ si kweli na maswali yenye } \\
\text { majibu mengi ya kuchagua kutoka kwa ni } \\
\text { pamoja na kauli zisizo kweli ambazo zinaweza } \\
\text { kuwakanganya wanaojitolea. } \\
\text { - Kauli za kweli/ si kweli zinaelekea kuwa na } \\
\text { majibu mengi ya "kweli" } \\
\text { - Mtindo wa kweli/ si kweli haujulikani katika } \\
\text { mazingira mengi; wanaojitolea wanaweza } \\
\text { kuzingatia kuwa ni ukosefu wa adabu kusema } \\
\text { kuwa kauli ambayo mshauri ametoa ni "kosa" }\end{array}$ \\
\hline $\begin{array}{l}\text { Mitazamo ya } \\
\text { kuelezea ubora } \\
\text { (maswali wazi) }\end{array}$ & $\begin{array}{l}\text { - Ilihimiza watu kuzungumza } \\
\text { kwa uwazi zaidi, uliza } \\
\text { maswali, na kudhihirisha } \\
\text { wazi zaidi kama taarifa } \\
\text { imeeleweka vyema. } \\
\text { - Inaruhusu mshiriki kujibu } \\
\text { kwa maneno yake } \\
\text { mwenyewe katika } \\
\text { mazungumzo yanay } \\
\text { olingana na moyo wa } \\
\text { idhini fahamishi. } \\
\text { - Wafanyikazi wanaweza } \\
\text { kupata hisi kama mshiriki } \\
\text { anaweza kutumia taarifa } \\
\text { kuamua kama ashiriki } \\
\text { katika jaribio na kufuata } \\
\text { itifaki. }\end{array}$ & $\begin{array}{l}\text { - Tathmini ni ya kiima; inahitaji wafanyikazi wawe } \\
\text { na uamuzi wa hali ya juu na kutofautiana kidogo } \\
\text { kimaana juu ya kiwango cha ufahamu wa } \\
\text { anayejitolea. } \\
\text { - Inahitaji mafunzo mapana ya wafanyikazi na } \\
\text { usaidizi wenye kuendelea. } \\
\text { - Inahitaji muda mwingi wa mfanyikazi } \\
\text { kutekeleza. } \\
\text { - Shabaha za kujiandikisha zinaweza kusababisha } \\
\text { kutokuwa na motisha kwa wafanyikazi } \\
\text { kubainisha kuwa anayejitolea haelewi taarifa } \\
\text { barabara vya kutosha kujiandikisha. }\end{array}$ \\
\hline
\end{tabular}




\section{Kiambatisho 2: Jaribio la kikliniki la HPTN 035: Orodha kaguzi ya kiwango cha ufahamu wa kujiandikisha katika idhini fahamishi, Tafsiri ya 1.0 (Imedondoolewa)}

Jaribio la HPTN 035 linatumia ukadiriaji wa maswali wazi ambao unaakisi elementi zinazohitajika za idhini fahamishi kulingana Kanuni za Shirikisho ya Marekani. Kila moja ya vipengele hivi pia ina elementi mahususi zaidi. Kuna za lazima mtu kujua na ambazo lazima yajibiwe kwa njia sawa na washiriki ili kusajiliwa.

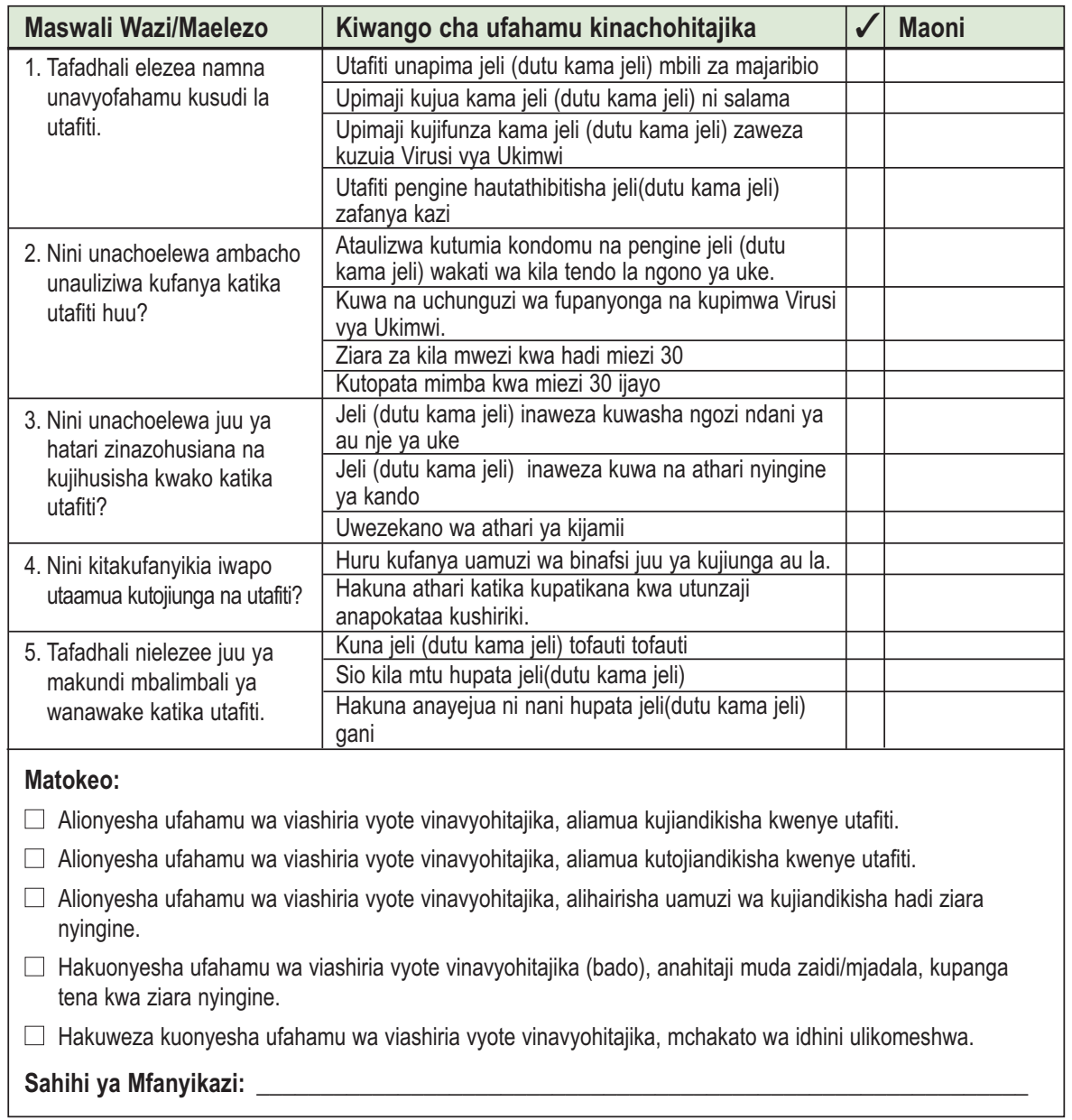

Asili: Coletti, A.S. 2005. "HPTN 035 informed consent comprehension assessment." Wasilisho katika warsha ya Population Council/Family Health International juu ya majaribio ya Idhini Fahamishi katika Uzuiaji wa Virusi vya Ukimwi, New York, 16-18 Mei. 
